WORLD VIEW

\title{
Risk factors for age related cataract in a rural population of southern India: the Aravind Comprehensive Eye Study
}

\author{
P K Nirmalan, A L Robin, J Katz, J M Tielsch, R D Thulasiraj, R Krishnadas, R Ramakrishnan
}

Br J Ophthalmol 2004;88:989-994. doi: 10.1136/bjo.2003.038380

Series editors: W V Good and S Ruit

See end of article for authors' affiliations

Correspondence to:

Correspondence to:
Alan L Robin, MD, 6115

Falls Road, STE 333 ,

Baltimore, MD 21209 -

2226, USA;

glaucomaexpert@cs.com

Accepted for publication 26 February 2004
Aim: To determine risk factors for lens opacities and age related cataract in an older rural population of southern India.

Methods: A cross sectional population based study of 5150 people aged 40 years and above from 50 clusters from three districts in southern India. The lens was graded and classified after dilation using LOCS III system at the slit lamp for cataract. Definite cataract was defined as nuclear opalescence $\geqslant 3.0$ and/or cortical cataract $\geqslant 3.0$ and/or PSC $\geqslant 2.0$.

Results: Definite cataracts were found in 2449 (47.5\%) of 5150 subjects and the prevalence of cataract increased with age. The age adjusted prevalence of cataract was significantly lower in males $(p=0.0002)$. Demographic risk factors - increasing age and illiteracy - were common for the three subtypes of cataract; females were more likely to have cortical cataracts and nuclear cataracts. Additionally, nuclear cataracts were associated with moderate smoking (OR:1.28, 95\% Cl:1.01 to 1.64), lean body mass indices (OR: 1.37, $95 \% \mathrm{Cl}: 1.17$ to 1.59 ) and higher waist to hip ratios (OR: $0.67,95 \% \mathrm{Cl}: 0.54$ to 0.82 ); cortical cataracts with hypertension (OR: $1.3995 \% \mathrm{Cl}: 1.11$ to 1.72), pseudoexfoliation (OR:1.53,95\% Cl:1.17 to 2.01), and moderate to heavy smoking; and posterior subcapsular cataracts with diabetes (OR:1.55, 95\% $\mathrm{Cl}: 1.12$ to 2.15 ), lean body mass (OR:1.32, 95\% Cl:1.11 to 1.57), and high waist to hip ratios (OR: 0.77, $95 \% \mathrm{Cl}: 0.62$ to 0.94$)$.

Conclusions: Risk factors for age related cataract in this population do not appear to be different from those reported in other populations. Further studies are required to identify the reason for the high prevalence of age related cataract and to understand better the role of each risk factor for cataractogenesis in this population.
A ge related cataract is the major cause of blindness in India. ${ }^{12}$ The prevalence of age related cataract and cataract surgery is higher in this rural southern Indian population than reported for most other populations studied. ${ }^{3}$ A previous study reported an earlier age of onset for age related cataract among people from the Indian subcontinent compared to other populations. ${ }^{4}$ Despite the public health significance of cataract in India, there are few reports on the risk factors for age related cataract from India. ${ }^{5-7}$ The role of environmental and personal risk factors for the development of age related cataract in this population is uncertain and may differ from that of white populations if earlier onset is indeed the case. Preventing or delaying the formation of cataract may help reduce the surgical burden on the eye care system of India. Identifying potential risk factors for age related cataract may further our understanding their role in cataractogenesis. This may help target higher risk populations for screening programmes, and develop appropriate preventive strategies. This paper reports on certain risk factors for age related cataract in a rural population aged 40 years and above in southern India.

\section{SUBJECTS AND METHODS}

The study design and methodology are described in previous publications. $^{38}$ To summarise, we randomly identified 50 representative clusters from three southern districts of the state of Tamil Nadu in southern India through multistage cluster sampling. Villages of less than 350 people were excluded from the list of villages for selection since they would not produce at least 100 subjects aged 40 and older, and would not be representative of the general population of these three districts. An additional consideration was the need to conserve resources and minimise lengthy travel to very small villages where there would be very few eligible subjects. Demographic details of eligible respondents in the selected clusters were collected by trained social workers after a door to door enumeration. All subjects aged 40 years and above were invited to the base hospital for a comprehensive ocular examination. Three levels of informed consent were used in this study, community, household, and individual. Before both screening and definitive examinations, the study was explained in detail to all potential participants and their voluntary consent was solicited. We insured that all subjects thoroughly understood the informed consent. We did not obtain thumbprints as this was problematic because many villagers could not read. All informed consent was thus verbally obtained, as a significant proportion of this population is illiterate. The study was approved, and annually reapproved, by the committee on human research at the Johns Hopkins Bloomberg School of Public Health and by the ethics review committee of the Aravind Eye and Children's hospitals. This investigation adhered to the tenets of the Declaration of Helsinki.

Trained social workers enumerated residents of households in the selected villages between 1994 and 1996. ${ }^{8}$ These workers were trained at Aravind Eye Hospital, Madurai, for data collection using the instruments developed for the study before the onset of the main study. Such training included collection of information from patients presenting to the hospital, staff at the hospital, and from people residing in villages near the hospital in a simulated study environment. In the field, workers collected demographic and other

Abbreviations: BMI, body mass index; LOCS, Lens Opacities Classification System; PSC, posterior subcapsular cataract; PXF, pseudoexfoliation 
information on medications and personal history for subjects aged 40 years or older before any ocular examinations. All subjects aged 40 and older were transported to the Aravind Eye Hospitals in either Madurai or Tirunelveli for a comprehensive ophthalmological examination that included measurements of presenting and best corrected visual acuity, subjective retinoscopic refraction, automated full threshold visual fields for subjects with best corrected visual acuity better than 6/60 using the C-24-2 full threshold program on the Humphrey 650 visual field analyser (Dublin, CA, USA), evaluation of pupillary response, external and anterior segment examination at the slit lamp biomicroscope, measurement of intraocular pressure with a Goldmann applanation tonometer (three independent readings in each eye), and gonioscopy using a Goldmann lens. After these examinations, pupils were dilated using either $1 \%$ tropicamide and/or 10\% phenylephrine unless otherwise contraindicated by gonioscopy. Subjects who had gonioscopic evidence of narrow angles and whom we felt were at risk for an attack of acute angle closure glaucoma had a laser peripheral iridectomy before dilatation of the pupils. We graded the lens at the slit lamp using the Lens Opacities Classification System (LOCS) III. $^{9}$ The standard set of photographs were mounted next to the slit lamp for grading. We recorded the presence and degree of nuclear opalescence and colour, cortical and posterior subcapsular cataracts. We graded nuclear cataracts with reference to standard photographs on a decimal scale of 0.1-6.9 based on optical density without reference to lens colour. Cortical opacity was graded on a decimal scale of 0.1-5.9 according to the opacity that obscured the light reflex on retroillumination. Posterior subcapsular cataract (PSC) was graded according to the estimated area of posterior capsule involved. Measurements of the greatest vertical and horizontal dimensions of the PSC were made and graded on a decimal scale of 0.1-5.9. A team of ophthalmologists standardised to each other and to a gold standard examiner graded the lens. Study ophthalmologists were standardised to the lens grading on three separate occasions: once immediately before the onset of the study, and at 6 and 12 months into data collection. The ophthalmologists were standardised between themselves as well as to a senior ophthalmologist considered the gold standard. We considered a weighted kappa score of less than 0.75 as requiring re-standardisation of the ophthalmologists with respect to lens grading. For this analysis, we defined cataract as nuclear opalescence $\geqslant 3.0$ and/or cortical cataract $\geqslant 3.0$ and/or PSC $\geqslant 2.0$.

\section{Assessment of potential risk factors}

Potential risk factors assessed as part of this study included a history of smoking, diabetes, hypertension, body mass index, waist to hip ratios, and pseudoexfoliation (PXF).

We defined systemic hypertension as either a measured systolic blood pressure $\geqslant 160 \mathrm{~mm} \mathrm{Hg}$ and/or a diastolic blood pressure $\geqslant 90 \mathrm{~mm} \mathrm{Hg}$ or current use of systemic antihypertensive medications. We have used this definition so that we can better compare our results to those of other studies on eye diseases. We measured the blood pressure of each study participant with a mercury column sphygmomanometer (Diamond Co Industrial Electronics and Allied Products, Electronics Cooperative estate, Pune, Maharashtra, India) using a standardised technique. Subjects were rested at least 5 minutes in a seated position before measuring the blood pressure. All blood pressure measurements were made on the left arm of each study subject, using a cuff of appropriate size at the level of the heart. The radial pulse was felt and the cuff level inflated $30 \mathrm{~mm} \mathrm{Hg}$ above the level at which the radial pulse disappeared and deflated slowly. The first and fifth Korotkoff sounds were recorded as systolic and diastolic blood pressure respectively. Diabetes was defined in this study as a measured postprandial blood sugar of $\geqslant 180 \mathrm{mg} / \mathrm{dl}$ or current use of blood sugar lowering medications. We measured the height of subjects using a measuring tape and weight using platform scales. We used height and weight measurements of individual subjects to calculate body mass index (BMI), which was defined as weight in kilograms/ height in metres squared. We classified subjects as lean if the BMI was $<20$ for males and $<19$ for females; as normal if the BMI was between 20-25 for males and between 19-24 for females; as overweight if the BMI was between 25-30 for males and between 24-29 for females; and obese if the BMI was $>30$ for males and $>29$ for females. We elicited a history of smoking; data regarding the number of cigarettes smoked per day was elicited for both current and past smokers. For this analysis, we categorised cigarette smoking as nonsmokers (never smoked), mild (up to 25th percentile of person years smoked), moderate (25-75th percentile), and heavy ( $>75$ th percentile). We did not, however, elicit a history for passive smoking or for pipe smoking (pipe smoking is extremely rare in this population). We measured waist and hip measurements for subjects to derive the waist to hip ratios. We categorised the waist to hip ratios as normal if they were $\leqslant 0.8$ for females and $\leqslant 1.0$ for males. ${ }^{10}$

We diagnosed PXF by the presence of typical white deposits on the anterior lens surface and/or iris; additional sites where we found PXF included the cornea, anterior vitreous face, posterior capsule, and even intraocular lens in cataract operated eyes. Before dilatation, we looked for PXF deposits on the corneal endothelium, iris, and iris margins using a detailed high magnification slit lamp assessment. After dilation, we examined the anterior lens surface using a narrow slit lamp beam under full illumination and high magnification to scan the lens left to right focusing on detecting early signs of PXF including pregranular radial lines as well as established granular deposits. We also looked for changes in the angle using gonioscopy including increased pigmentation, PXF deposition, and PXF material within the angle.

\section{Statistical analysis}

We used Stata statistical software version 7.0 (College Station, TX, USA) for analysing data. We selected the eye with the highest cataract score for statistical analysis in people who had no previous cataract surgery. We used the phenotype of the cataract in the eye with the worst score for analysis in a person with cataract in both eyes; and in the eye with cataract for a person with cataract in one eye and no cataract in the fellow eye. We assumed the worst score for an operated eye if a person had previous cataract surgery in one eye with cataract in the fellow eye. Because we did not have access to surgical records that could give us information as to cataract phenotype at surgery, we analysed this subgroup separately with those subjects operated on for cataract in both eyes. We performed bivariate and multivariate analyses by subject to look for associations between cataract and potential risk factors using multiple logistic regression models separately for nuclear, cortical, posterior subcapsular, and mixed cataracts; all variables were included in the model as categorical variables We used decades of years to adjust for age in our analysis. We assessed interactions between the different variables in the multiple logistic regression models and considered interactions to be significant if the $p$ value was $<0.05$. Prevalence estimates, odds ratios (OR), and $95 \%$ confidence intervals (95\% CI) are presented. Confidence intervals for prevalence estimates and odds ratios from the regression analyses were calculated taking into account design effects (deff) associated with cluster sampling using generalised estimation equation. 


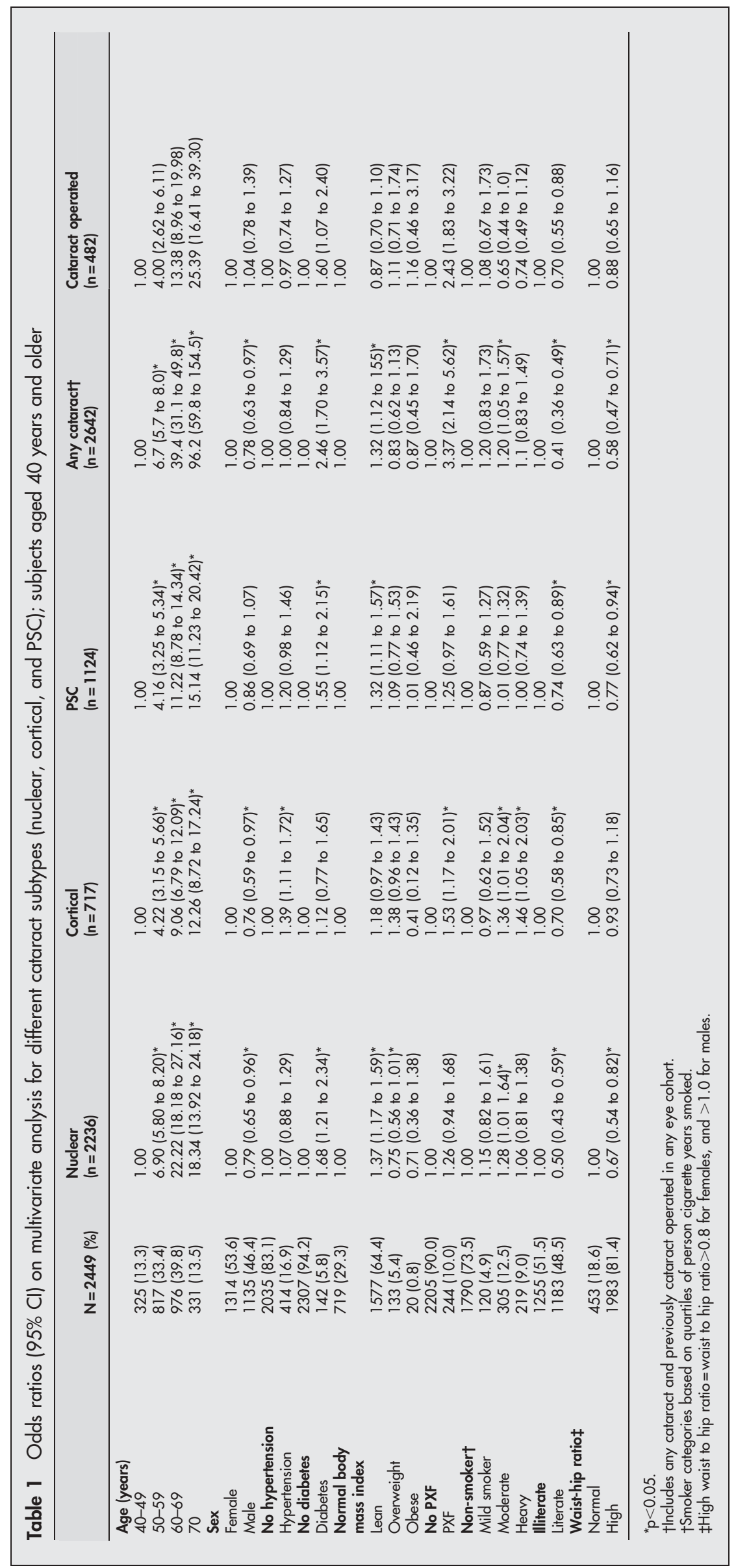




\section{RESULTS}

Definite cataract in one or both eyes was present in 2499 $(47.5 \%)$ of 5150 the subjects. The age adjusted prevalence (adjusted to US population estimates for the year 2000) of definite cataract in this population was 61.9\% (95\% CI: 60.6 to 63.3 ), indicating that our population had a younger age structure than that of the United States. In those eyes with cataracts, nuclear cataract $(n=2236,43.5 \%)$ was the most common type of cataract; cortical cataract was present in 717 (13.9\%), posterior subcapsular cataract in $1024(19.9 \%)$, and mixed cataracts were present in 1167 (47.7\%).

The prevalence of definite age related cataracts of all types increased significantly $(\mathrm{p}<0.001)$ with increasing age, from $15.7 \%$ among those aged $40-49$ years to $79.4 \%$ among those aged $\geqslant 70$ years. The odds ratio $(95 \% \mathrm{CI})$ for definite cataract for age groups 50-59, 60-69, and $\geqslant 70$ (reference category $40-$ 49 years) were 1.3 ( 1.1 to 1.4 ), 4.3 (3.7 to 5.0 ), and 3.8 (3.0 to $4.8)$, respectively. After adjusting for age, males had lower odds (odds ratios $0.8,95 \% \mathrm{CI}: 0.7$ to 0.9 , deff $=1.0$ ) for cataract than females. The odds ratio for cataract was significantly lower for literate subjects (OR: 0.5, 95\% CI: 0.4 to 0.6 , deff $=2.5$ ), after adjusting for age and sex.

Cataract surgery in one or both eyes had been performed for $482(9.4 \%)$ people including 153 people who had bilateral cataract surgery $(31.7 \%$ of all subjects having had cataract surgery). The prevalence of cataract surgery in either eye increased with increasing age $(\mathrm{p}<0.001)$.

The most common location for cortical lens opacities was the lower nasal quadrant ( $\mathrm{n}=799,57.2 \%)$. Even among 235 eyes with minimal cortical changes between 0 to 1 LOCS III grade, the most common location was the lower nasal $(\mathrm{n}=130,55.3 \%)$, and lower temporal segment $(\mathrm{n}=95$, $40.4 \%)$.

We found hypertension (OR 1.3, 95\% CI: 1.1 to 1.6 , deff $=1.4)$, diabetes $($ OR $1.9,95 \%$ CI: 1.4 to 2.6 , $\operatorname{deff}=1.2)$, and PXF (OR 4.6, 95\% CI: 3.7 to 5.6, deff $=0.5$ ) to be associated with cataract on bivariate analysis. Cataract was associated with subjects with lean body mass (OR: 1.7, 95\% CI: 1.4 to 2.1 , deff $=3.1$ ) on bivariate analysis. We found a protective effect for cataract (OR: $0.8,95 \%$ CI: 0.6 to 0.9 , deff $=0.8$ ) among those who never smoked on bivariate analysis. We found a protective effect for cataract among those with higher waist to hip ratios (OR:0.8, 95\% CI: 0.7 to 0.9 , $\operatorname{deff}=4.1$ )

There were no significant interactions between variables in the multiple logistic regression models. Multivariate analysis for nuclear cataract showed an association with increasing age, sex, illiteracy, moderate smoking, lean and overweight body mass indices, and a higher waist to hip ratio (table 1). Multivariate analysis for cortical age related cataract showed an association with increasing age, female sex, illiteracy, moderate and heavy smoking, hypertension, and PXF. Multivariate analysis for PSC showed an association with increasing age, illiteracy, diabetes, lean body mass indices, and high waist to hip ratios.

\section{DISCUSSION}

The lack of adequate epidemiological information on risk factors for age related cataract in India makes it difficult to determine if the large magnitude of cataract is essentially due to an increased predisposition to cataracts in this population, or entirely due to inadequate surgical rates. Such information is essential to develop preventive strategies even as eye care programmes in India are beginning to improve capacity in order to deal with the huge backlog without compromise on quality. Our results do not suggest the presence of environmental or personal risk factors for age related cataract peculiar to this population that have not been reported from other large population based studies. ${ }^{11-19}$ We also did not find any occupational link to cataract formation as over $90 \%$ of our population were primarily agricultural workers. ${ }^{8}$

Our study was not designed to prospectively evaluate details pertaining to nutritional intake and ultraviolet light exposure. To assess ultraviolet light exposure as a risk factor for cataract, ocular exposure measurements should be taken, and we did not have the resources to obtain such measurements in this study. We intentionally chose not to use a questionnaire as most questionnaires are not able to capture this information with accuracy primarily owing to the high potential for recall bias. Using a questionnaire in this specific illiterate and agrarian population probably would not have provided us with valid data. The alternate of measuring serum nutrient levels is also a one time measure that may not give a true picture of the nutritional status of a person over time, and may not be reflective of the possible nutritional stresses on the lens that produce cataract. We are limited by the absence of antioxidant measurements and dietary history to explore if there are differences in nutritional status that can explain the higher prevalence of age related cataract in this population.

The association of lower body mass index with increased risk for cataract has also been previously reported from India. ${ }^{4}$ Moderate calorie intakes has been shown to delay the formation of cataract in animal studies, the proposed biological mechanism being lower energy intake that prolongs the antioxidant or proteolytic capabilities of the lens. ${ }^{20}$ BMI also affects glucose levels; higher glucose levels are associated with a higher risk for cataract. ${ }^{21}$ It is possible that the association of cataract with lower BMI is confounded by lower socioeconomic status, diarrhoeal diseases, or micronutrient deficiency. It is possible that the biological mechanisms that associate BMI with cataract are similar with respect to waist to hip ratios. In models where both BMI and waist to hip ratios were present, low BMI and waist to hip ratios were independently associated with increased risk of cataract, indicating that there may be several nutritional pathways to increased cataract risk.

It is not clear if the nutritional deficiency status precedes cataract formation or possibly accelerates cataract progression, or is a consequence of the reduced vision associated with cataracts that has a bearing on the earning potential of the person, and consequently food intake. It is possible that

\begin{tabular}{|c|c|c|}
\hline Risk factor & Relative risk & Population attributable risk (\%) \\
\hline Hypertension & 1.14 & 2.11 \\
\hline Diabetes & 1.36 & 1.59 \\
\hline Smoking & 1.02 & 0.62 \\
\hline Lean body mass index & 1.32 & 15.78 \\
\hline
\end{tabular}


there may be nutritional stresses over the life period of people in the Indian subcontinent that predisposes them to cataracts and may also possibly explain the earlier onset of cataracts in this population. The earlier and higher prevalence of cataracts among migrant Indians compared to the local population reported from United Kingdom ${ }^{22}$ suggests that factors other than the environment including nutrition or genetic factors may be involved in cataractogenesis for people from the Indian subcontinent.

Previous studies have reported lower educational levels associated with higher prevalence of age related cataract. ${ }^{41-13}$ We found similar results with illiteracy associated with age related cataract of any type. The biological association between education and cataracts is not clear. It is possible that low education is confounded by several variables including exposure to sunlight, hygiene, illnesses, and nutritional factors among others. Previous studies have reported lower surgical coverage for people with less education in this population. ${ }^{23} 24$

We found moderate to heavy smoking associated with nuclear and cortical cataracts in our study. A previous clinic based case-control study from India, however, reported no association between smoking and age related cataract. ${ }^{25}$ It is not clear if this difference between the two studies is related to study populations-ours was a population based sample. The difference may also be due to a possible dose-response relation relating to differences in type of cigarettes used and numbers smoked per day between urban and rural residents. We are unable to determine if this is indeed so as our study population was entirely rural in character. Previous population based studies have also reported an association between smoking and cataracts, especially nuclear cataracts. ${ }^{16-19}$

We explored the possibility of PXF as a risk factor for age related cataract as both PXF and cataracts are age related. ${ }^{26}$ We found PXF to be significantly associated with cortical cataracts in our study population. The association between cortical cataracts and UV-B light exposure has been reported previously ${ }^{27}$; however, our study was not designed to explore this possible association. We, instead, looked at the location of cortical changes as a possible indicator of an association with UV-B radiation in this population. We found cortical changes to be most commonly located in the inferior-nasal quadrant supporting a possible association with UV-B radiation. This finding concurs with previous studies in the United States and elsewhere. ${ }^{28} 29$ To explore this association further, we also looked at the location of minimal cortical changes (cortical changes $<1.0$ ); we found these changes to be more in the inferior nasal quadrant. We do not believe that this is because of ease of view by the examiners as all pupils were widely dilated, making it unlikely that any superior opacities would be hidden by the upper eyelid.

We found that posterior subcapsular cataracts were relatively common in this population. We do not feel that this is a result of examiner error or bias as all examiners were well versed in using the LOCS III instrument and were continuously standardised

It is possible that the higher prevalence of age related cataracts in India is entirely attributable to the low surgical coverage. $^{23} 30$ However, the prevalence of cataract surgery among Indians aged more than 40 years is reported to range from $9.5 \%$ to $13.7 \%$ compared to $3.8 \%$ for a population in the same age group in Melbourne, Australia. ${ }^{31-33}$ We do not think that the lower odds of having a cataract for males is the result of the higher surgical rate for males. We have previously found a comparable surgical rate for males and females in this population. ${ }^{3}$

Smoking remains a modifiable risk factor for age related cataracts in this rural southern Indian population, along with changes in lifestyle including maintaining an optimal body mass index and waist to hip ratios that reduce the risks for diabetes and/or systemic hypertension (see table 2). Lean body mass indices were more probably associated with cataracts than obese body mass indices. It is therefore not surprising that higher waist to hip ratios are protective for cataract, although our study was not designed to determine any biological basis for this observation. Even though the regression model implies there are associations with lifestyle variables, the attributable risks are not large. The largest attributable risk was for lean body mass but since the direction of the association is unclear, these risk factors do not hold much promise for controlling the cataract problem.

\section{ACKNOWLEDGMENTS}

This study was supported in part by unrestricted grants from Allergan, Inc, Alcon Laboratories, Inc, and Zeiss Corp.

\section{Authors' affiliations}

P K Nirmalan, R D Thulasiraj, R Krishnadas, R Ramakrishnan, Aravind Medical Research Foundation, Aravind Eye Care System, Madurai, Tamilnadu, India-625020

A L Robin, J Katz, J M Tielsch, Department of Ophthalmology, and Department of International Health, Johns Hopkins Bloomberg School of Public Health, Baltimore, MD, USA

J Katz, J M Tielsch, Dana Center for Preventive Ophthalmology, Johns Hopkins University Schools of Medicine and Public Health, Baltimore, MD, USA

\section{REFERENCES}

1 Thylefors B, Negrel AD, Pararajasegaram R, et al. Global data on blindness. Bull World Health Organ 1995;73:115-21.

2 Mohan M. Survey of blindness-India (1986-1989), summary results. New Delhi: Programme for the Control of Blindness, Ministry of Health and Family Welfare, Government of India, 1992.

3 Nirmalan PK, Krishnadas R, Ramakrishnan R, et al. Lens opacities in a rural population of southern India. The Aravind Comprehensive Eye Study. Invest Ophthalmol Vis Sci 2003;44:4639-43.

4 Chatteriee A, Milton RC, Thyle S. Prevalence and aetiology of cataract in Punjab. Br J Ophthalmol 1982;66:35-42.

5 Mohan M, Sperduto RD, Angra SK, et al. India-US case control study of age related cataracts. Arch Ophthalmol 1997;115:105-11.

6 Badrinath SS, Sharma T, Biswas J, Srinivas V. A case control study of senile cataract in a hospital based population. Indian J Ophthalmol 1996:44:213-17.

7 Ughade SN, Zodpey SP, Khanolkar AV. Risk factors for cataract: a case control study. Indian J Ophthalmol 1998;46:221-7.

8 Thulasiraj RD, Nirmalan PK, Ramakrishnan R, et al. Blindness and vision impairment in a rural southern Indian population: the Aravind Comprehensive Eye Survey. Ophthalmology 2003;110:1491-8.

9 Chylack LT, Wolfe JK, Singer DM, et al. The Lens Opacities Classification System III. Arch Ophthalmol 1993;111:831-6.

10 Bjorntorp P. Classification of obese patients and complications related to the distribution of surplus fat. Am J Clin Nutr 1987;45:1120-5.

11 Hiller R, Sperduto RD, Ederer F. Epidemiologic associations with cataract in the 1971-1972 National Health and Nutrition Examination Survey. Am J Epidemiol 1983; 118:239-49.

12 Kahn HA, Leibowitz HM, Ganley JP, et al. The Framingham Eye Study. II. Association of ophthalmic pathology with single variables previously measured in the Framingham Heart Study. Am J Epidemiol 1977;106 33-41.

13 Hiller R, Sperduto RD, Ederer F. Epidemiologic associations with nuclear, cortical, and posterior subcapsular cataracts. Am J Epidemiol $1986 ; 124: 916-25$.

14 Italian-American Cataract Study Group. The risk factors for age-related cortical, nuclear and posterior subcapsular cataracts. Am J Epidemiol $1991 ; 133: 541-3$

15 Leske MC, Chylack LT Jr, Wu S-Y, The lens opacities case-control study group.. The lens opacities case control study. Risk factors for cataract. Arch Ophthalmol 1991;109:244-51

16 West S, Munoz BE, Emmett EA, et al. Cigarette smoking and risk of nuclear cataracts. Arch Ophthalmol 1989;107:1166-9.

17 Klein BEK, Klein R, Linton KLP, et al. Cigarette smoking and lens opacities. The Beaver Dam Eye Study. Am J Prev Med 1993;9:27-30.

18 Hankinson SE, Willett WC, Colditz GA, et al. A prospective study of cigarette smoking of cigarette smoking and risk factor of cataract surgery in women. JAMA 1992;268:994-8.

19 Christen WG, Manson JE, Seddon JM, et al. A prospective study of cigarette smoking and risk of cataract in men. JAMA 1992;268:989-93.

20 Taylor A, Zuliani AM, Hopkins RE, et al. Moderate caloric restriction delays cataract formation in the Emory mouse. FASEB J 1989;3:1741-6. 
21 Harding JJ, van Heyningen R. Epidemiology and risk factors for cataract. Eye 1987; 1:537-41.

22 Thompson JR. The demand incidence of cataract in Asian immigrants to Britain and their descendants. Br J Ophthalmol 1989;73:950-4

23 Nirmalan PK, Padmavathi A, Thulasirai RD. Sex inequalities in cataract blindness burden and surgical services in south India. Br J Ophthalmol 2003;87:847-849

24 Nirmalan PK, Thulasiraj RD, Maneksha V, et al. A population based eye survey of older adults in Tirunelveli district of south India: blindness, cataract surgery and visual outcomes. $\mathrm{Br} J$ Ophthalmol 2002;86:505-12

25 Mohan M, Sperduto RD, Angra SK, et al. India-US case control study of age related cataracts. Arch Ophthalmol 1989;107:670-6.

26 Ritch R, Schlotzer-Schrehardt U. Exfoliation syndrome. Surv Ophthalmol 2001:45:265-315.

27 West SK, Valmadrid CT. Epidemiology of risk factors for age-related cataract. Surv Ophthalmol 1995;39:323-34.
28 Schein OD, West S, Munoz B et al. Cortical lenticular opacification: distribution and location in a longitudinal study. Invest Ophthalmol Vis Sci 1994; 35:363-6.

29 Sasaki H, Kawakami Y, Ono M, et al. Localization of cortical cataract in subjects of diverse races and latitude. Invest Ophthalmol Vis Sci 2003:44:4210-14.

30 Murthy GVS, Gupta S, Ellwein LB, et al. A population based eye survey of older adults in a rural district of Rajasthan: 1. Central vision impairment, blindness and cataract surgery. Ophthalmology 2001;108:679-85.

31 Bachani D, Gupta SK, Murthy GVS, et al. Visual outcomes after cataract surgery and cataract surgical coverage in India. Int Ophthalmol 1999;23:49-56

32 Dandona L, Dandona R, Naduvilath TJ, et al. Population based assessment of the outcome of cataract surgery in an urban population of southern India. Am J Ophthalmol 1999;127:650-8.

33 McCarty CA, Nanja MB, Taylor HR. Operated and unoperated cataract in Australia. Clin Exp Ophthalmol 2000;84:22-7.

\section{Video reports}

To view the video reports in full visit our website www.bjophthalmol.com and click on the link to video reports.

- Penetrating ocular injury due to a fish hook: surgical removal. SD M Chen, D Chiu, C K Patel

- Retinal ganglion cell axon response to guidance molecules. S F Oster, D W Sretavan

- Marin-Amat syndrome. A Jogiya, C Sandy

- Excision of subcutaneous dirofilariasis of the eyelid D Mallick, T P Ittyerah

- Thixotropy: a novel explanation for the cause of lagophthalmos after peripheral facial nerve palsy. M Aramideh, J H T M Koelman, P P Devriese, F VanderWerf, J D Speelman

- Surgical revision of leaking filtering blebs with an autologous conjunctival graft. K Taherian, A Azuara-Blanco

- Dipetalonema reconditum in the human eye. THuynh, J Thean, R Maini

- Evaluation of leucocyte dynamics in mouse retinal circulation with scanning laser ophthalmoscopy. H Xu, A Manivannan, G Daniels, J Liversidge, P F Sharp, J V Forrester, I J Crane

- An intraocular steroid delivery system for cataract surgery. D F Chang

- Pearls for implanting the Staar toric IOL. D F Chang

- Capsule staining and mature cataracts: a comparison of indocyanine green and trypan blue dyes. D F Chang

- Endoscopy to aid anterior segment surgery. J E Moore, A Sharma

- Reconstruction of the Ocular Surface in LOGIC Syndrome. J E Moore, V Kumar, $J$ R Ainsworth, S Shah

- A suture technique to manage a case of severe early flap displacement after laser in situ keratomileusis. L Spadea, P Pantaleoni, G Bianco 